\title{
Fractal Characteristics of Transverse Crack Propagation on CRTSII Type Track Slab
}

\author{
Yue-Lei He, Jian-Kang Shen (D), Zai-Wei Li, and Hong-Yao Lu \\ School of Urban Transportation, Shanghai University of Engineering Science, Shanghai 201620, China \\ Correspondence should be addressed to Jian-Kang Shen; sjkmaroon@163.com
}

Received 12 March 2019; Revised 25 May 2019; Accepted 16 June 2019; Published 25 July 2019

Academic Editor: Konstantinos Karamanos

Copyright (C) 2019 Yue-Lei He et al. This is an open access article distributed under the Creative Commons Attribution License, which permits unrestricted use, distribution, and reproduction in any medium, provided the original work is properly cited.

\begin{abstract}
Objectives of this study are to examine influence of the surface crackles fractal characteristics of CRTSII track slab on its propagation velocity and stress intensity factor (SIF) and to improve the service performance of track slab. In this paper, fractal dimension D of transverse crack on the surface of CRTSII track slab was calculated by image processing, digital image technology, and box-counting method. Based on the fractal structure of transverse crack on track slab surface, a fractal transverse crack propagation model was established. The impact of fractal effect on the surface crack propagation velocity and SIF of CRTSII track slab was analyzed quantitatively. The fractal dimension D of transverse crack is 1.0652 on the surface of CRTSII track slab. The actual measured crack propagation velocity $V_{0}$ on the surface of the track slab can be corrected as the true crack propagation velocity $V$. When the $V_{0} / C_{r}=0.899<1$, the SIF $K_{l}\left(L_{(1.0652, t)}, V\right)$ of transverse dynamic fractal crack propagation on the surface of track slab has been equal to 0 , and the $V_{0}$ is always lower than Rayleigh wave velocity. In addition, the true attenuation rate of SIF for CRTSII track slab surface transverse dynamic crack propagation is faster than the actual measured value.
\end{abstract}

\section{Introduction}

CRTSII type ballastless track structure as concrete slab structure, subjected to train dynamic load, temperature load, and other complex environments, and the track slab surface will inevitably show a large number of cracks. In order to study the crack propagation state and stability of reinforced concrete materials, the fracture mechanics of concrete were developed in the early stage. It is presumed that the material is based on the ideal linear elastic material and the stress state at the crack tip is calculated by linear elastic mechanics. Reference [1] aimed at the crack propagation problem of elastic-plastic solid; putting forward the crack tip opening displacement is invoked as the characteristic parameter to measure the crack growth and established the fracture criterion of crack under elastoplastic condition. On the basis of fracture mechanics theory, finite element analysis has been extensively used in the study of crack propagation characteristics with the development of computer technology. Reference [2] used finite element method (FEM) including cohesive zone model (CZM) to simulate cracking failure mechanisms. Based on fracture mechanics, a knowledge base is established on how FEM can be effectively applied to predict pavement performance against cracking failure mechanisms. Two main approaches are presented in this study; they are focused meshes and CZM. While focused meshes may be used to predict the number of cycles for the crack propagation, CZM may be used to study the controlling stresses and mechanisms responsible for crack propagation. Reference [3] reviewed the development and the application of the scaled boundary finite element method for fracture analysis. When the method applied to two-dimensional linear fracture mechanics, any kinds of stress singularities are represented analytically without local refinement, special elements, and enrichment functions. Coupling procedures with the extended finite element method (XFEM), meshless method and boundary element method to handle changes in the crack morphology have been established. These developments result in an efficient framework for fracture modelling. Reference [4] established the model of water-bearing crack propagation under the mortar layer of CRTSII slab ballastless track under train load. The pressure above the crack and the strength factor at the crack tip are calculated and analyzed by numerical method. 
All the above literature had played a role in promoting and reference, to study crack propagation characteristics of CRTSII type track slab surface and improve the maintenance strategy of track slab. However, much work has been done in the past to study the propagation of flat straight crack tip in homogeneous media. In fact, most materials are inhomogeneous media, and their deformation and failure are nonlinear processes under nonequilibrium conditions, and the geometric shape of crack propagation path is complex and irregular. The crack measured by experiment is essentially a fractal geometry, which greatly affects the fracture evolution process of materials [5]. Therefore, on the basis of fracture mechanics, Xie Heping [6] has gradually formed a complete theory-fractal fracture mechanics in the study of deformation and failure of heterogeneous materials. The influence of fractal crack propagation on stress intensity factor and crack propagation velocity was qualitatively and quantitatively explained.

According to the criterion of stress intensity factor (SIF) for crack growth, when the SIF at the crack tip is larger than the critical SIF, the crack will make unstable propagation [7]. Therefore, surface crack of CRTSII type track slab is affected by the stress concentration effect of its own tip, and the possibility of continuous instability propagation is very great [8]. This destruction is a gradual process with crack initiation, propagation, and series and develops in depth, forming cracks. After a certain degree of development, there will be interface debonding along the direction of the steel bar near the cracks, which will cause fatigue damage and service performance degradation of track slabs. Stress intensity factor (SIF) is an important index [9] which can effectively characterize the crack growth state, and its size is related to the crack propagation velocity in dynamic crack propagation. Therefore, it is of great significance to accurately calculate the propagation velocity and stress intensity factor of CRTSII type track slab surface cracks for measuring and controlling the fracture of CRTSII type track slab, improving the service performance of track slab. Our research group has proved that the extended path of the transverse crack of CRTSII type track slab has fractal characteristics [10], and combined with the theory of fractal fracture mechanics, it focused on the investigation of the following problems: whether the surface crack propagation velocity and stress intensity factor (SIF) of CRTSII type track slab are affected by fractal effect, and whether the real crack propagation velocity can be obtained by the relationship between them.

\section{Fractal Dimension of CRTSII Type Track Slab Transverse Crack}

2.1. Image Acquisition and Processing of Cracks. The authors used high magnification camera to capture surface cracks on a typical passenger dedicated line in East China and selected CRTSII type track slab surface transverse cracks as the research object. Figure 1 shows the crack propagation captured on site and the obtained crack length is $1900 \mathrm{~mm}$. In addition, with the help of image processing technology and digital image technology [11-13], the image region of the main crack path was extracted, and the binarization was carried out. The obtained crack is converted into a two-dimensional black-and-white digital image ( 0 for black, 1 for white), as shown in Figure 2.

2.2. Fractal Dimension of Crack. In fractal theory, as a very important parameter, fractal dimension can quantitatively describe the irregular, fractional, and fragmented physical phenomena (fractal dimension reflects the validity of space occupied by complex bodies, which is a measure of irregularity of complex structures. It is a quantity without units). For the fractal structure in nature, box dimension as an approximate value of fractal dimension is widely used and can be used to obtain many valuable results $[14,15]$. According to the calculation principle of box dimension, the fractal dimension of the transverse crack of CRTSII type track slab surface is approximated by the box-counting method [16]. The boxcounting method is a numerical procedure for estimating approximately the fractal dimension of a geometrical pattern. Such method considers the properties of space-filling of the geometrical pattern as complexity indicator (autosimilitude). By means of such approach, the crack is covered by $\mathrm{N}$ boxes, and the number of the boxes of a particular size $r$ is computed for estimating how many of such boxes are necessary to cover the whole cracks. As the size $\mathrm{r}$ is close to 0 , according to Falconer [17], this relationship can be written as

$$
D=\lim _{r \rightarrow 0}\left[\frac{-\log N(r)}{\log (r)}\right]
$$

where $N(r)$ represents the number of grids covering the crack in the image; $r$ is the length of the mesh. The total number $N$ of meshes covering the cracks necessarily varies with the change of $r$. According to the above formula, when $r$ is sufficiently small, we have

$$
N(r)=\beta * r^{-D}
$$

where $\beta$ is a constant. Equation (2) can be converted into the following relationship:

$$
\ln \mathrm{N}=\ln (\beta)-D \ln (r)
$$

In the equation, fractal dimension $\mathrm{D}$ of the crack propagation path on the surface of CRTSII type track slab is the absolute of the straight line slope which can be obtained by least square method fitting the new data point $(\ln (r), \ln N)$. The two-dimensional digital image of the processed CRTSII type track slab surface transverse crack is a numerical matrix of $\omega \times h$ pixels, where $\omega$ is width of the image; $h$ is length of the image. Therefore, the mesh side length $r$ is under a minimum size of 1 pixel, which is the limit of the meshing size; and the maximum size is the image width $\omega$. We aimed to achieve the purpose of using grids to equally divide images and minishing pixel points. In this study, the binarization image width $\omega$ of transverse crack on the surface of CRTSII type track slab was increased to $h$ [18]. Figure 3 presents the schematic diagram of the obtained data from box-counting method. 


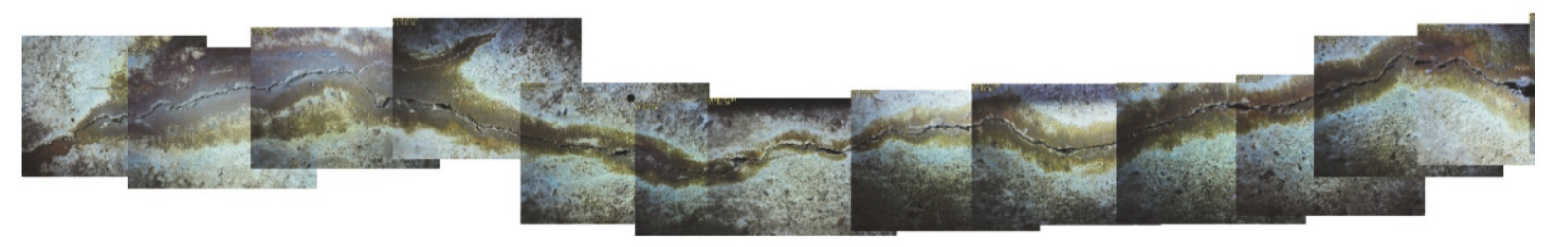

FIGURE 1: Transverse crack propagation on the surface of CRTSII type track slab.

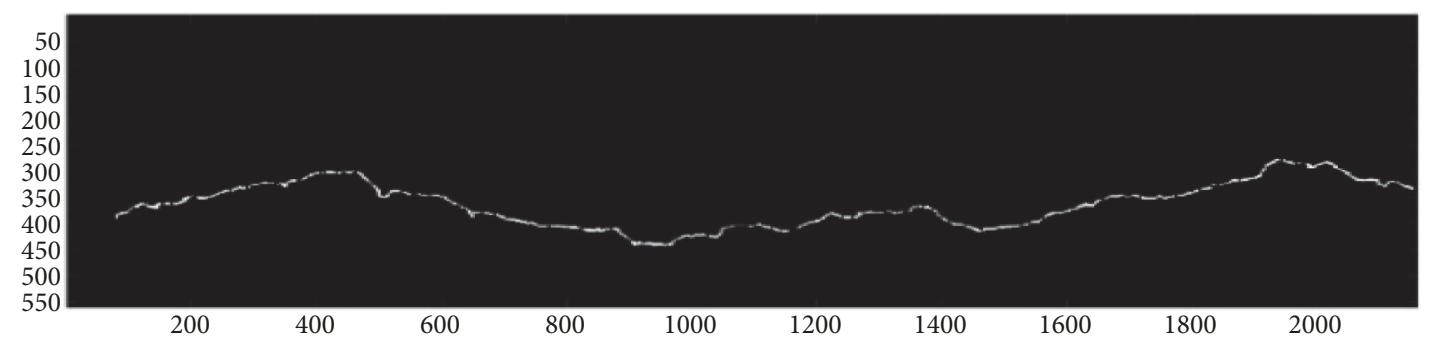

FIGURE 2: Binarization of transverse crack in CRTSII type track slab surface.

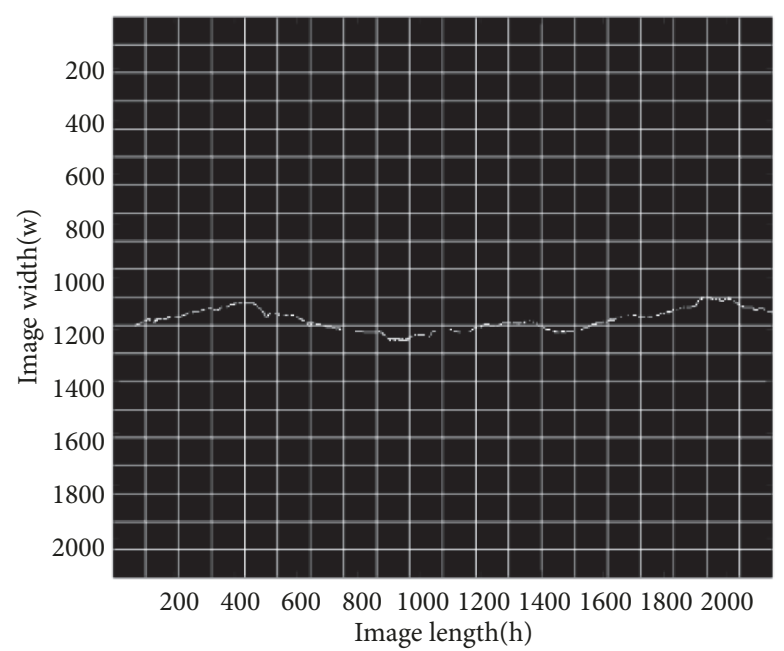

Figure 3: Schematic diagram of transverse crack fractal boxcounting method for slab surface.

The fractal dimension D of CRTSII track slab surface transverse crack is approximated by box-counting method. The result is shown in Figure 4(d).

In Figure 4(c), the actual box-counting shows the number of boxes occupied by cracks; the space-filling box-counting represents the number of boxes covered by the whole picture.

Figure 4(a) shows the 0 and 1 of the initial binarization images are inverted and used as input data. Figure 4(b) shows relationship between the number of boxes and box size $r$, and the number of boxes covered decreases as the box size increases. In Figure 4(c), by comparing actual box-counting and space-filling box-counting, we can see that, under the same box size $r$, the number of actual boxes is always lesser than that of space-filling boxes. As shown in Figure 4(d), new data points $(\ln (r), \ln N)$ have been regressed and obtained the straight line equation as follows:

$$
\mathrm{Y}=8.4282-1.0652 \mathrm{X}
$$

Because the reliability of the regression equation is unknown, $F$ test is used to test the significance of the regression coefficient and sets the significance level at $\alpha=$ 0.01 ; the test results are as follows.

It can be seen that the F-statistic for the linear regression model of the variables $\ln (r)$ and $\ln N$ corresponds to 992.855 , and the significance probability $\mathrm{P}$ is lower than the significance level of 0.01 . Therefore, the influence of the variable $\ln (r)$ on $\ln N$ is significant, and the regression equation has reliability. The results show that the fractal dimension $\mathrm{D}$ of CRTSII type track slab surface transverse crack studied in this paper is 1.0652, and it has a fractal structure.

\section{Transverse Crack Propagation Velocity}

Past investigations show that even brittle materials can also produce rough fracture surfaces and irregular propagation paths, and many researchers adopted different theoretical models to analyze these curvilinear or bending crack propagation [19], where the fractal crack propagation model can successfully describe the crack propagation characteristics [20]. Therefore, based on the known fractal characteristics of CRTSII type track slab transverse cracks, a fractal crack propagation model was established by taking into account the surface transverse cracks of track slab. Figure 5 shows relationship between actual measurement and true crack propagation status.

In Figure $5, L_{0}, L(\delta)$ are, respectively, crack propagation length or the incremental crack step $\Delta \alpha$ and the fractal (real) crack propagation length measured by scaling $\delta$ in equal time; $V_{0}, V$ are, respectively, actual measurement and true 


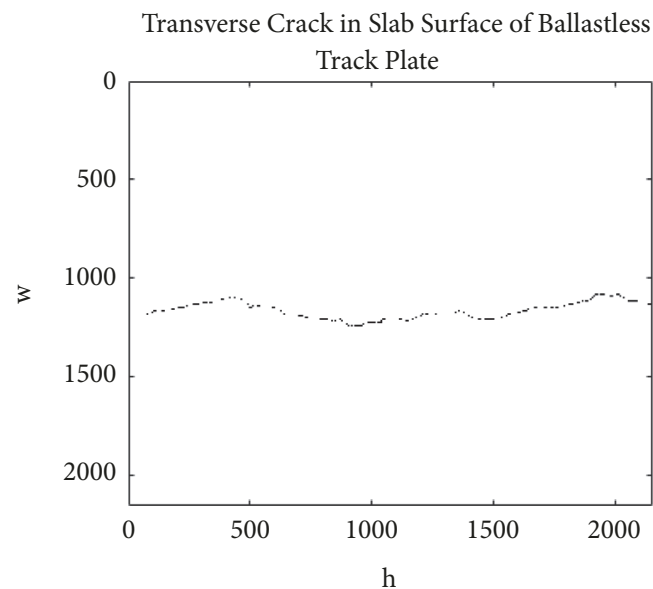

(a)

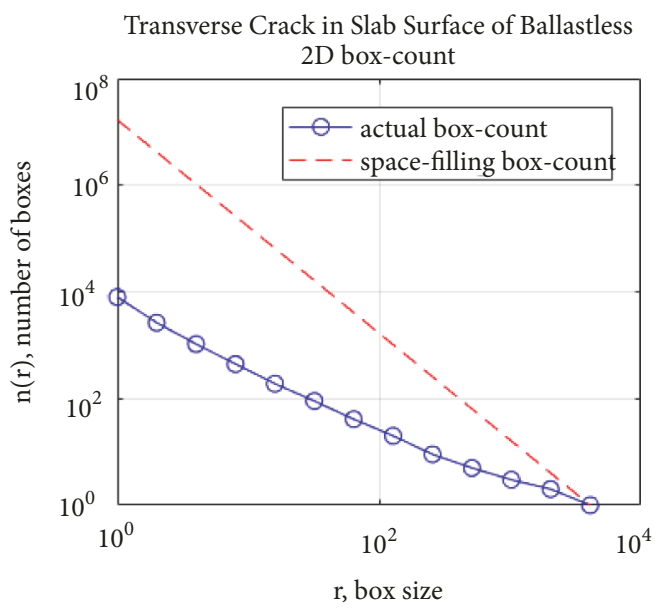

(c)
Transverse Crack in Slab Surface of Ballastless

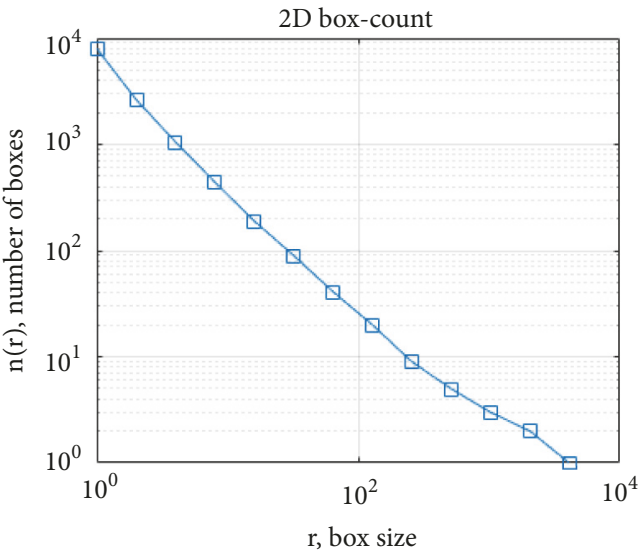

(b)

Transverse Crack in Slab Surface of Ballastless

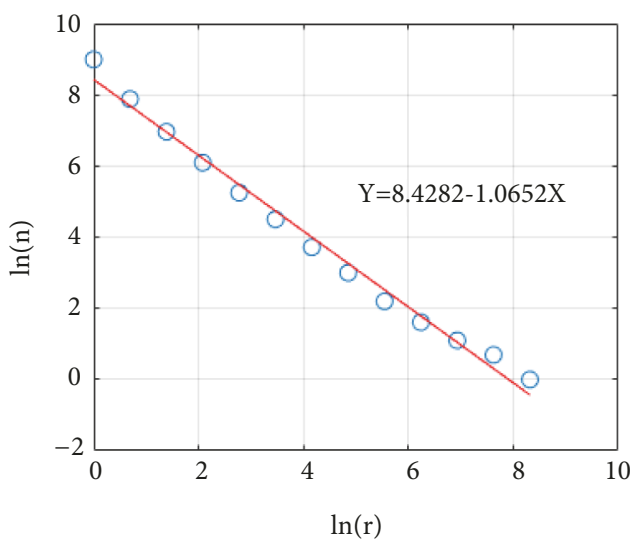

(d)

FIGURE 4: Calculation of crack fractal dimension D by box-counting method. (a) Reversing binarization input graph; (b) the number of boxes varied curve with $r$; (c) comparison diagram of actual and space-filling box-counting; (d) the regression straight line equation of data points $(\ln (r), \ln N)$.

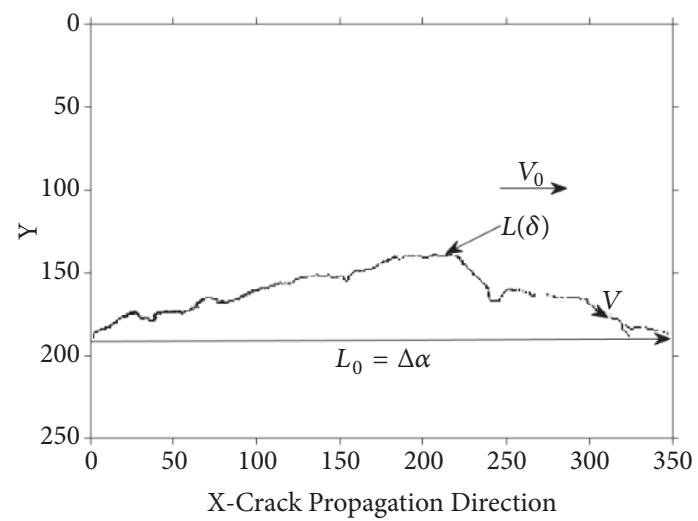

FIGURE 5: Transverse fractal crack propagation model of CRTSII type track slab surface.

crack propagation velocity; $X$. The main crack propagation direction. When $\delta \longrightarrow 0$, the transverse crack propagation length $L(\delta)$ and $\delta$ of the CRTSII type track slab satisfy the following relationship:

$$
L(\delta)=\lim _{\delta \rightarrow 0} N(\delta) \delta
$$

where $N(\delta)$ denotes the number of segments obtained by measuring the transverse crack length of track slab surface with a ruler $\delta$. According to (5), the actual propagation length of transverse fractal cracks on track slab surface increases with the decease of $\delta$, that is, $L(\delta) \longrightarrow \infty$ when $\delta \longrightarrow$ 0 . However, in natural fractals, there is a scale upper limit, also called scale invariance [21]. Therefore, length of the transverse crack of the CRTSII type track slab also has a range of scale invariance. That is $\delta \in\left[\delta_{\min }, \delta_{\max }\right]$, where $\delta_{\min }$, is determined by the microstructure of material. CRTSII type track slab is a concrete structure mixed with different raw materials. Therefore, it is needed to determine the suitable $\delta_{\text {min }}$ according to the actual situation. In this paper, a high magnification camera is used to photograph, sample and digital image analysis the cracks on the surface of track slab 
[22], as shown in Figure 6. It can be observed in the diagram that the cracks mainly propagate along the cementitious material (cement, additives mixture) in Figures 6(a) and $6(\mathrm{~b})$, and only a small number of penetrating aggregate phenomenon (black wire frame).

According to the self-similarity (fractal characteristics) of the surface transverse crack of CRTSII type track slab, the actual crack propagation length $L(D, t)$ is determined by fractal theory. The equation is as follows:

$$
L(D, t)=L_{0}^{D} \delta^{1-D}
$$

where $L(D, t)>L_{0}$. Measurement results [23, 24] shows that the grain size can be used as the minimum roughness scale for irregular crack propagation, and the crack propagation modes of materials depend on the size of crystal inclusions, and the microstructural characteristics influence the crack propagation modes of materials. Therefore, in the case of combining the raw material particle size distribution of CRTSII track slab. Therefore, in the case of combining the raw material particle size distribution of CRTSII track slab. In this study, the minimum value of $\delta_{\min }$ is $0.02 \mathrm{~mm}$ (cement fineness); that is to say, using $\delta=\delta_{\min }=0.02 \mathrm{~mm}$ to approximate the $\mathrm{C} 55$ concrete minimum particle D of CRTSII type track slab. Therefore, the surface roughness of transverse cracks on CRTSII type track slab can be expressed as

$$
\frac{L(D, t)}{L_{0}}=\left(\frac{\delta}{L_{0}}\right)^{1-D}
$$

when the incremental step $\Delta \alpha=L_{0}$ of the track slab transverse crack in the "macroscopic" propagation direction, and the fractal crack growth velocity $V$ is assumed to be constant in this increment within the same time. The equation is as follows:

$$
\frac{\Delta \alpha}{V_{0}}=\frac{L(D, t)}{V}
$$

Substituting (7) and $\delta=0.02 \mathrm{~mm}$ into (8) yields

$$
\begin{aligned}
& \frac{V}{V_{0}}=\left(\frac{\Delta \alpha}{\delta}\right)^{D-1} \\
& \frac{V}{V_{0}}=(50 \Delta \alpha)^{D-1}
\end{aligned}
$$

This study is based on the transverse fractal crack growth model on the surface of CRTSII type track slab, and it is assumed that the numerical value of increment steps $\Delta \alpha$ of crack growth can be actually measured in equal time. Thus, the power exponential relation between the velocity ratio $V / V_{0}$ and the fractal dimension $\mathrm{D}$ can be obtained by substituting the known $\Delta \alpha$ into (10). Table 2 shows the power exponent relationship between the track slab transverse crack propagation velocity ratio $V / V_{0}$ and $D$ under different incremental steps $\Delta \alpha$ (Assumed value). When $\mathrm{D}=1.0652$, the ratio of fractal propagation velocity of CRTSII type track slab surface transverse crack to measure propagation velocity is shown in Table 2
According to Table 1, the curves of the fractal crack growth velocity and the actually measured crack growth velocity ratio $V / V_{0}$ with the fractal dimension $\mathrm{D}$ under different incremental step lengths were obtained, and when $D=1.0652$, the change curve of the velocity ratio with the increment step size $\Delta \alpha$. As shown in Figure 7.

From Figure 7(a), it can be observed that the fractal propagation velocity $V$ of CRTSII type track slab transverse crack is greater than the measured crack growth velocity $V_{0}$. When the fractal dimension $D$ of crack is $1.15-1.20$, the fractal propagation velocity $V$ of local crack is about twice that of measured crack velocity $V_{0}$. The relationship between $V / V_{0}$ and incremental step $\Delta \alpha$ shows that when the crack increment is the smaller, the speed ratio $V / V_{0}$ is the closer to 1 . That is to say, when the measuring time of crack propagation increment on track plate surface is shortened, the smaller the actual measured crack growth increment, the closer the measured crack growth velocity $V_{0}$ to the true crack growth velocity $V$ (theoretical velocity value). Therefore, when the fractal dimension $D$ of the CRTS II type tracks slab and the measured crack increment $\Delta \alpha$ are known, they will be substituted into the following formula:

$$
V=(50 \Delta \alpha)^{D-1} * V_{0}
$$

Crack propagation velocity $V_{0}$ of the actual track slab can be corrected as the true crack propagation velocity $V$ using (11).

\section{SIF of Crack Propagation}

Depending on the theory of fracture mechanics, the stress intensity factor of crack growth can effectively characterize the state of crack growth and its stability. In order to study the effect of fractal effect on the stress intensity factor (SIF) of crack growth, XIE Heping [6] established a fractal interpolation model of crack growth trajectory based on fractal interpolation theory. According to Freund's dynamic fracture theory, when the interpolation time $\Delta t \rightarrow 0$, the stress intensity factor at the crack tip motion $L_{(t)}$ can be approximated as the stress intensity factor at the crack tip motion $L_{(D, t)}$. Therefore, the fractal interpolation method is used to generalize Freund's dynamic stress intensity factor to describe the motion of fractal crack tip. That is,

$$
\begin{aligned}
K\left(L_{(D, t)}, V\right) & =h(V) K\left(L_{(D, t)}, 0\right) \\
h(V) & =\frac{\left(1-V / C_{r}\right)}{\left[1-\left(V / C_{r}\right)\left(C_{r} / C_{\delta}\right)\right]^{1 / 2}}
\end{aligned}
$$

where $K\left(L_{(D, t)}, V\right)$ and $K\left(L_{(D, t)}, 0\right)$ are dynamic and static fractal crack growth stress intensity factors, respectively; $h(V)$ is a universal function of crack propagation velocity; $C_{\delta}$ and $C_{r}$ are elastic expansion wave speed and Rayleigh wave velocity, where $C_{r} / C_{\delta}$ can be approximated to

$$
\frac{C_{r}}{C_{\delta}}=\frac{(0.862+1.14 v)}{\left\{(1+v)[2(1-v) /(1-2 v)]^{1 / 2}\right\}}
$$



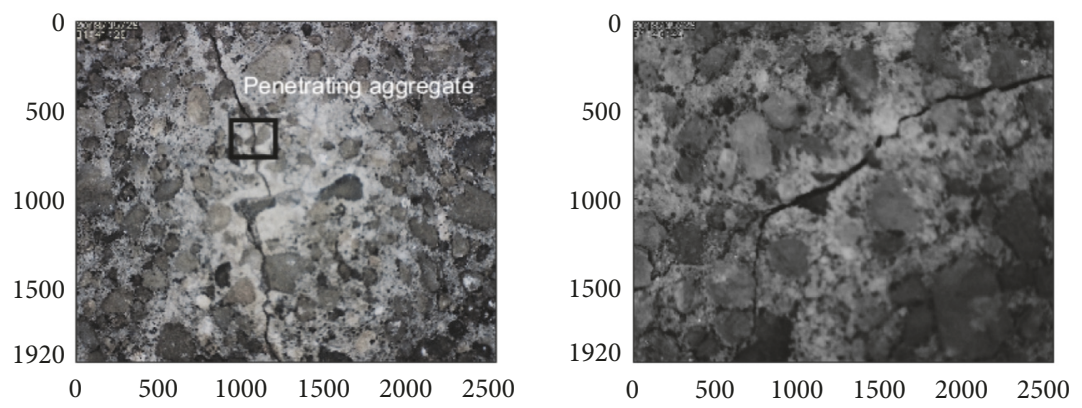

(a)
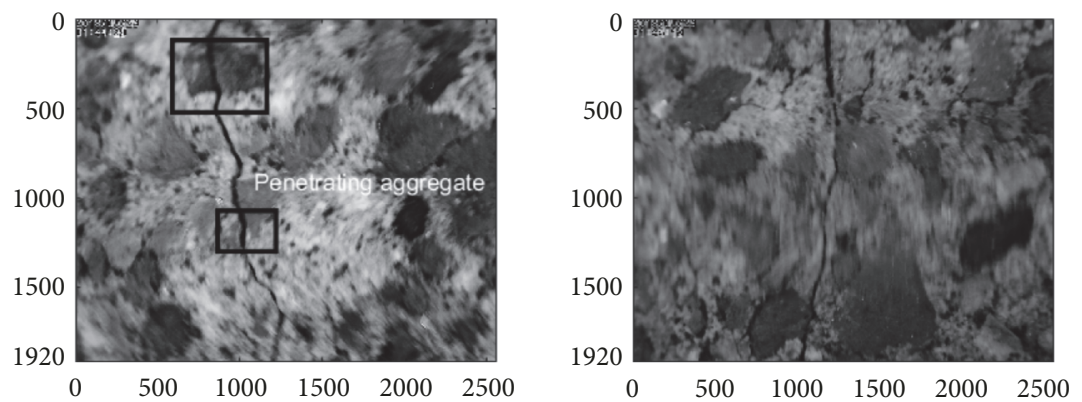

(b)

FIGURE 6: Crack specimen of CRTSII type track slab surface: (a) upper surface of track slab; (b) side surface of track slab.

TABLE 1: Calculated typical parameter data from $\mathrm{F}$ test.

\begin{tabular}{lcccc}
\hline Correlation Coefficient R & F-Statistic & Significant Probability P & Slope & Intercept \\
\hline 0.995 & 992.855 & 0.000 & -1.0652 & 8.4282 \\
\hline
\end{tabular}

where $v$ is the Poisson's ratio of the structure. By substituting (9) and (13) into (12), the ratio of stress intensity factors for dynamic and static fractal crack propagation can be obtained.

$$
\frac{K\left(L_{(D, t)}, V\right)}{K\left(L_{(D, t)}, 0\right)}=\frac{\left[1-\left(V_{0} / C_{r}\right)(\Delta \alpha / \delta)^{D-1}\right]}{\left[1-\left(V_{0} / C_{r}\right)\left(C_{r} / C_{\delta}\right)(\Delta \alpha / \delta)^{D-1}\right]^{1 / 2}}
$$

Fractal dimension $\mathrm{D}=1.0652$, Poisson's ratio $v=0.2$, and minimum dimension $\delta=0.02$ of CRTSII type track slab surface transverse cracks as known parameters substitute into (14) and (15). The stress intensity factor ratio of CRTSII track slab surface transverse dynamic and static fractal crack growth can be obtained.

$$
\frac{K_{l}\left(L_{(D, t)}, V\right)}{K_{l}\left(L_{(D, t)}, 0\right)}=\frac{\left[1-1.29\left(V_{0} / C_{r}\right)(\Delta \alpha)^{0.0652}\right]}{\left[1-0.718\left(V_{0} / C_{r}\right)(\Delta \alpha)^{0.0652}\right]^{1 / 2}}
$$

If the crack increment is assumed to be $\Delta \alpha=0.2 \mathrm{~mm}$ (of course, $\Delta \alpha$ can also take other values, $\Delta \alpha$ is the smaller, and the relative equation (17) is the more accurate), the relationship between the stress intensity factor ratio of the surface dynamic and static fractal crack growth of CRTSII track slab and $V_{0} / C_{r}$ can be obtained.

$$
\frac{K_{l}\left(L_{(D, t)}, V\right)}{K_{I}\left(L_{(D, t)}, 0\right)}=\frac{\left[1-1.162\left(V_{0} / C_{r}\right)\right]}{\left[1-0.646\left(V_{0} / C_{r}\right)\right]^{1 / 2}}
$$

Therefore, stress intensity factor ratio $K_{l}\left(L_{(D, t)}, V\right) /$ $K_{,}\left(L_{(D, t)}, 0\right)$ of dynamic and static fractal crack propagation of CRTSII type track slab surface with the change curve of $V_{0} / C_{r}$ is shown in Figure 8.

It can be observed in the diagram that when $D=1$ does not consider the fractal effect of track slab surface crack propagation, combined with (15), only when $V_{0} / C_{r}=1$, the stress intensity factor ratio of CRTSII type track slab surface transverse dynamic and static crack propagation is equivalent to 0 . That is, the stress intensity factor $K_{l}\left(L_{(1, t)}, V\right)$ of dynamic crack propagation is equal to 0 . However, under the influence of fractal effect, when $V_{0} / C_{r}=0.899<1$, the stress intensity factor $K_{1}\left(L_{(1.0652, t)}, V\right)$ of CRTSII type track slab surface transverse dynamic fractal crack propagation can be already equal to 0 . At the same time, the surface transverse crack propagation of CRTSII track slab is influenced by fractal effect, and the measured crack propagation velocity $V_{0}$ is always lower than that of Rayleigh wave velocity $C_{r}$. According to the stress intensity factor criterion of crack propagation, after transverse crack initiation on CRTSII track slab surface, the crack propagation velocity increases gradually, and the true stress intensity factor of dynamic crack 


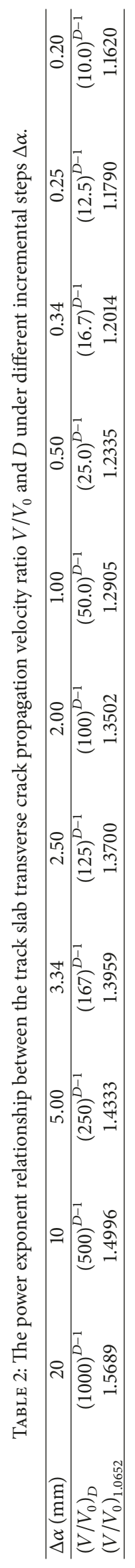




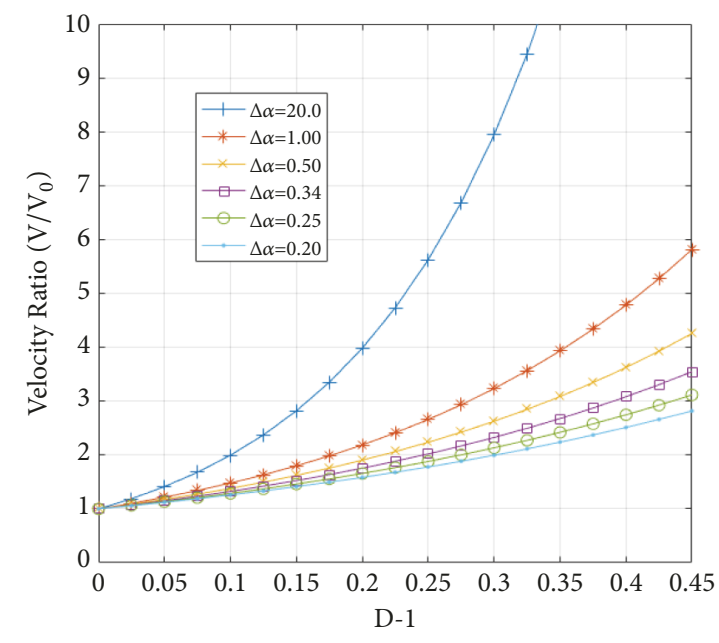

(a)

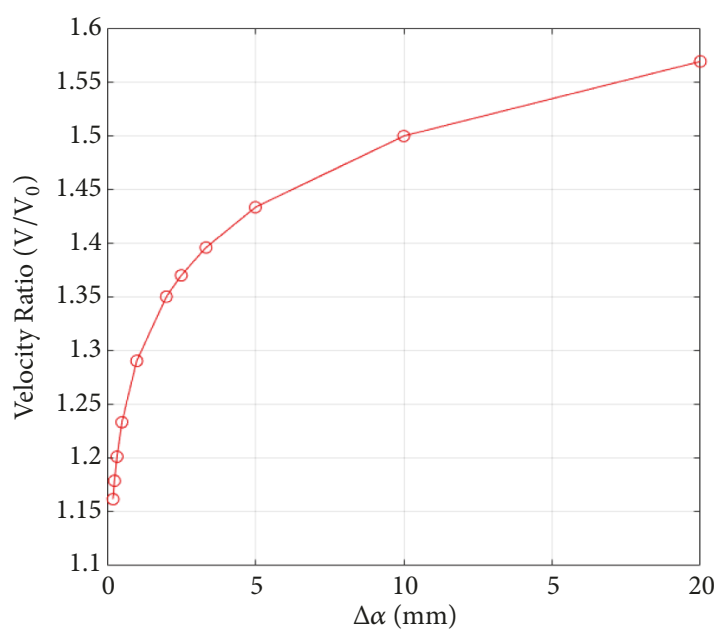

(b)

FIGURE 7: CRTSII type track slab surface transverse crack propagation: (a) the change curve of $V / V_{0}$ with $D$; (b) the change curve of $V / V_{0}$ with $\Delta \alpha$.

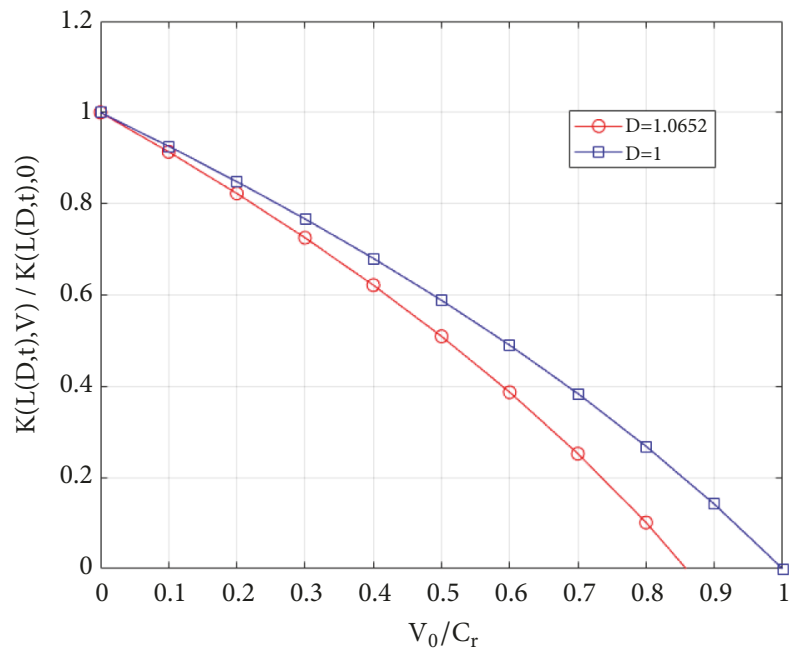

Figure 8: The SIF's ratio $\left(K_{,}\left(L_{(D, t)}, V\right) / K_{,}\left(L_{(D, t)}, 0\right)\right)$ of dynamic and static fractal crack propagation of CRTSII type track slab surface with the change curve of $V_{0} / C_{r}$.

propagation decreases faster than the actual measured value due to fractal effect.

\section{Conclusions}

This study has carried out the investigation and analysis of using digital image technology, box-counting method and fractal fracture mechanics. It is found that the method can effectively analyze the influence of fractal effect on the surface crack propagation velocity and stress intensity factor of CRTSII type track slab. On the basis of present study, the following conclusions can be drawn:

(a) CRTSII type track slab, as a concrete structure, is affected by the train dynamic load and its nonlinear temperature gradient for a long time. It appears more cracks in some track slab surface. The service performance of the track slab is dramatically reduced, leading to potentially dangerous issue to drive safety with the expansion and deepening of the crack. Therefore, it is critical to monitor and inhibit the surface crack propagation on the surface of CRTSII type track slab.

(b) Fractal dimension $\mathrm{D}$ of transverse crack on the surface of CRTSII type track slab is 1.0652 with a fractal structure. Actually measured crack propagation velocity $V_{0}$ of the surface transverse cracks of CRTSII type track slab is less than the fractal propagation velocity $\mathrm{V}$.

(c) When the measurement period of crack growth increment is shortened, the measured crack propagation velocity $V_{0}$ gradually approaches the true crack growth velocity $V$ (theoretical velocity value) with the decrease of the measured crack growth increment $\Delta \alpha$. The actual measured crack propagation velocity $V_{0}$ on the surface of the track slab can be corrected as the true crack propagation velocity $V$.

(d) Under the influence of fractal effect, when $V_{0} / C_{r}=$ $0.899<1$, stress intensity factor $K_{l}\left(L_{(1.0652, t)}, V\right)$ of transverse dynamic fractal crack propagation on the surface of CRTSII type track slab is equal to 0 . Meanwhile, the measured transverse crack propagation velocity $V_{0}$ of CRTSII type track slab surface is always lower than Rayleigh wave velocity. After transverse crack initiation on the surface of CRTSII track slab, the true stress intensity factor of dynamic crack propagation decreases faster than the actual measured value.

The fractal effect of crack propagation path has a significant effect on the crack propagation velocity and stress intensity factor of CRTSII type track slab. This study provides 
an alternative method for further investigation of fractal fracture mechanics. In addition, the proposed method can be used to monitor the fracture of CRTSII type track slab and to improve the understanding of service performance of track slab for engineers.

\section{Data Availability}

The data used to support the findings of this study are included within the article.

\section{Conflicts of Interest}

The authors declare that they have no conflicts of interest.

\section{Authors' Contributions}

The authors confirm contribution to the paper as follows: Yuelei He and Jiankang Shen contributed to study conception and design; Yuelei $\mathrm{He}$ and Jiankang Shen contributed to data collection; Yuelei He and Jiankang Shen contributed to analysis and interpretation of results; Zaiwei Li and Hongyao Lu contributed to draft manuscript preparation. All authors reviewed the results and approved the final version of the manuscript.

\section{Acknowledgments}

The authors would like to acknowledge National Natural Science Foundation for Youth Project of China (51808333) and Key Supporting Projects of Shanghai Municipal Science and Technology Commission (16030501400) for their support.

\section{References}

[1] A. A. Wells, "Application of fracture mechanics at and beyond general yelding," British Welding Jounal, vol. 10, pp. 563-570, 1963.

[2] M. A. Elseifi, J. Baek, and N. Dhakal, "Review of modelling crack initiation and propagation in flexible pavements using the finite element method," International Journal of Pavement Engineering, vol. 19, no. 3, pp. 251-263, 2017.

[3] C. Song, E. T. Ooi, and S. Natarajan, "A review of the scaled boundary finite element method for two-dimensional linear elastic fracture mechanics," Engineering Fracture Mechanics, vol. 187, pp. 45-73, 2018.

[4] X. Guihong, L. Xueyi, Y. Rongshan et al., "Effect of train load and water coupling to crack extension of CRTSII track," Journal of Railway Engineering Society, vol. 36, no. 10, pp. 76-80, 2014 (Chinese).

[5] G. P. Cherepanov, A. S. Balankin, and V. S. Ivanova, "Fractal fracture mechanics-A review," Engineering Fracture Mechanics, vol. 51, no. 6, pp. 997-1033, 1995.

[6] X. Heping, Introduction to Fractal-Rock Mechanics, Science Press, Beijing, China, 1996.

[7] C. Jin and Z. Shushan Fracture Mechanics, Beijing, China, 2012.

[8] T. Sain and J. Chandrakishen, "Probabilistic assessment of fatigue crack growth in concrete," International Journal of Fatigue, vol. 30, no. 12, pp. 2156-2164, 2008.
[9] J. De, Q. Qin, and L. Changan, Numerical Calculation Method in Fracture Mechanics and Its Engineering Application, Science Press, Beijing, China, 2009.

[10] S. Yunfei, H. Yuelei, Z. Zhiyuan et al., "Char-acteristic analysis of transverse crack growth for CRTS II slab track based on image processing," Journal of Rail Science and Engineering, vol. 14, no. 10, pp. 2059-2064, 2017.

[11] K. R. Castleman, Digital Image Processing, Publishing House of Electronics Industry, Beijing, China, 2002.

[12] X. Yong, C. Qiang, S. Zhenguo et al., "Application of fractal theory in welding image processing," Journal of Image and Graphics, vol. 7, no. 1, pp. 86-90, 2002.

[13] V. K. Gadi, S. Bordoloi, A. Garg, L. Sahoo, C. Berretta, and S. Sekharan, "Effect of shoot parameters on cracking in vegetated soil," Environmental Geotechnics, vol. 5, no. 2, pp. 79-93, 2018.

[14] H. Yan, W. Taiyong, W. Jian et al., "Reseach on fractal box dimension anti-noise performance and its application in fault diagnosis," Chinese Journal of Scientific Instrument, vol. 32, no. 03, pp. 540-545, 1965.

[15] G. Zheng, J. Zhao, L. Li, X. Cheng, and M. Wang, "A fractal analysis of the crack extension paths in a Si3N4 ceramic tool composite," International Journal of Refractory Metals and Hard Materials, vol. 51, pp. 160-168, 2015.

[16] J. Carrillo and W. Avila, "Assessment of seismic damage of thin and lightly reinforced concrete walls using fractal dimension of cracking," Earthquake Engineering \& Structural Dynamics, vol. 64, no. 4, 2017.

[17] K. Falconer, Fractal Geometry. Mathematical Foundations and Apliccations, John Wiley \& Sons Ltd, Chichester, West Sussex, England, 2nd edition, 2003.

[18] P. Ruidong, X. Heping, and J. Yang, "Computation method of fractal dimension for 2-D digital image," Journal of China University of Mining \& Technology, vol. 01, pp. 22-27, 2004.

[19] R. V. Goldstein and J. Lewandowski, "Surface roughness induced attenuation and changes in the propagation velocity of long Rayleigh-type waves," Acta Mechanica, vol. 91, no. 3-4, pp. 235-243, 1992.

[20] X. Heping and D. J. Sanderson, "Fractal effects of dynamic crack propagation," Chinese Journal of Theoretical and Applied Mechanics, vol. 01, pp. 18-27, 1995.

[21] L. Shida and L. Shishi, Fractal in Physics, Peking Univesity Press, Beijing, China, 2014.

[22] C. Cherian and D. N. Arnepalli, "Material characterization by digital image analysis:a review," Environmental Geote-chnics, 2016.

[23] X. Heping, Damage Mechanics of Rock and Concrete, China University of Mining and Technology Press, Jiangsu, China, 1990.

[24] D. L. Turcotte, Fractals and Chaos in Geology and Geophysics, Cambridge University Press, Cambridge, UK, 1992. 


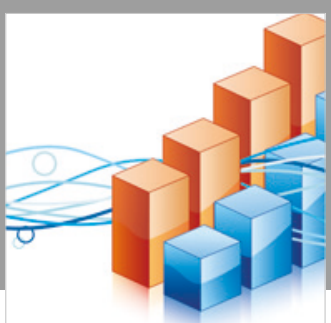

Advances in

Operations Research

\section{-n-m}
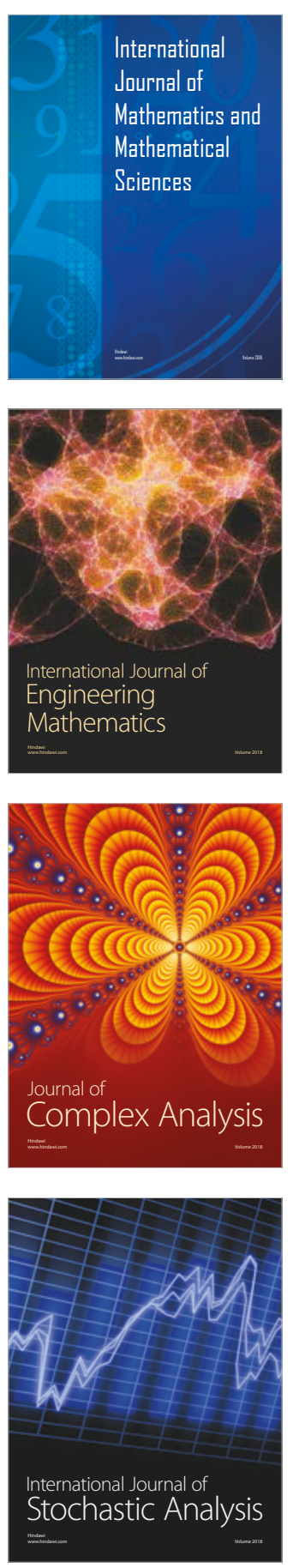
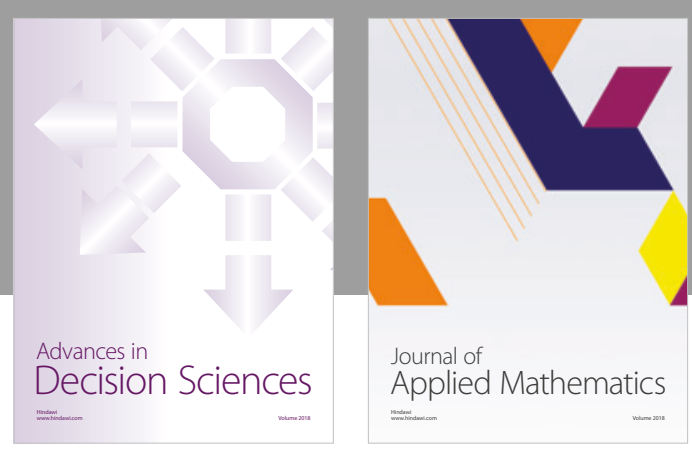

Journal of

Applied Mathematics
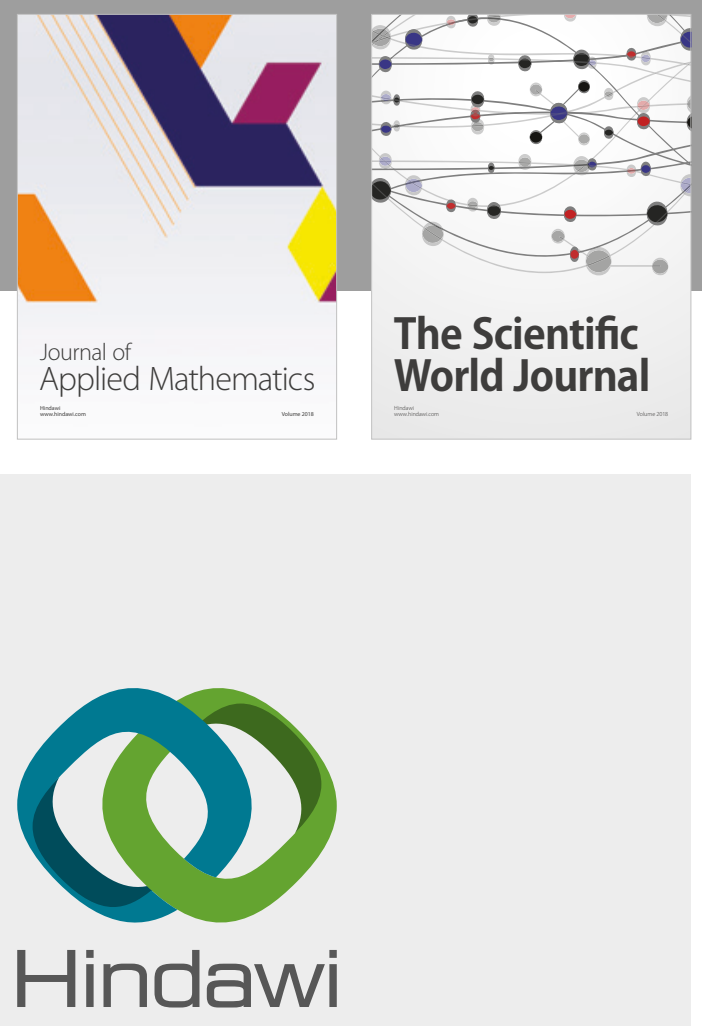

Submit your manuscripts at

www.hindawi.com

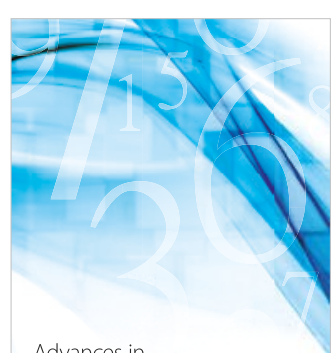

Advances in
Numerical Analysis
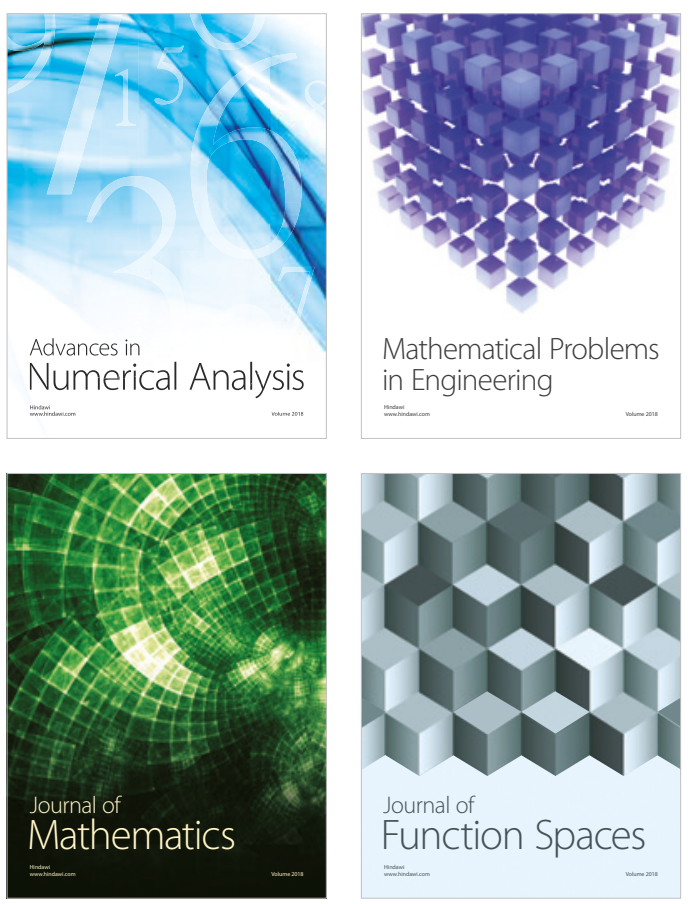

Mathematical Problems in Engineering

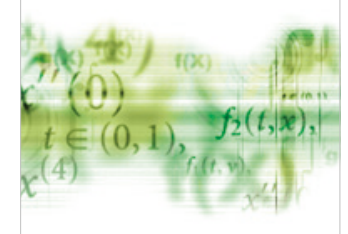

International Journal of

Differential Equations

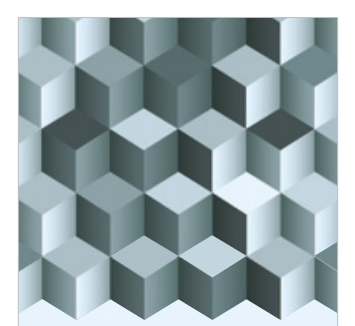

Journal of

Function Spaces

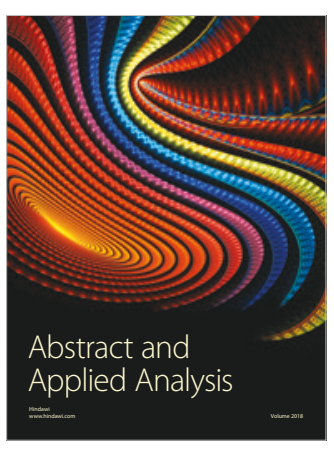

The Scientific

World Journal

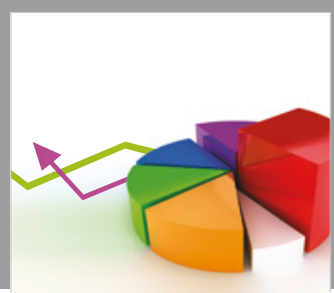

Journal of

Probability and Statistics
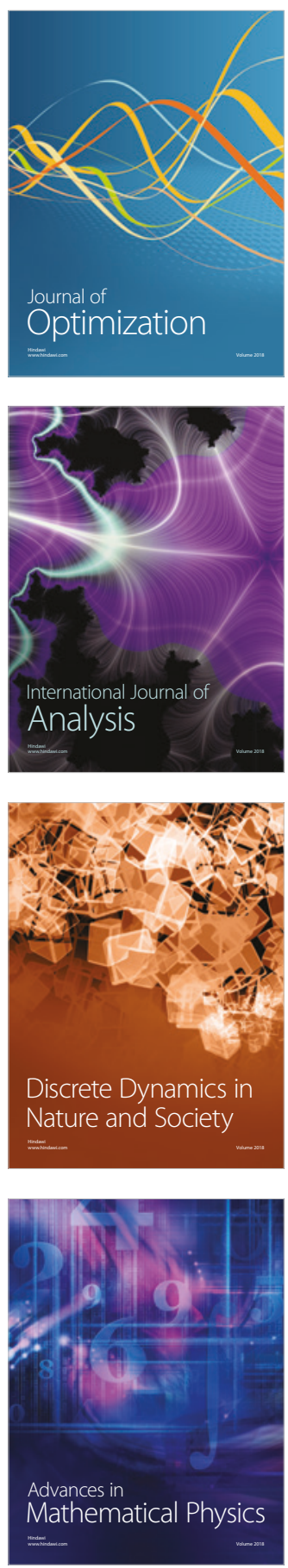